\title{
Intra-Frame Transmission Adaptation for Fast Fading MIMO-OFDMA Systems
}

\author{
Balkan Kecicioglu, Hlaing Minn, Senior Member, IEEE, and Chia-Chin Chong, Senior Member, IEEE
}

\begin{abstract}
We propose multiple antenna transmission methods for fast fading channel conditions. Proposed methods allocate alternative multiple antenna transmission modes depending on the SNR, modulation order and Doppler frequency to increase reliability, i.e., decrease bit-error-rate (BER). A major difference of our approach from previous works is to allow different transmission modes during a single frame by considering the channel variations within the frame for high mobility scenarios. First method effectively makes use of available channel knowledge by allocating beamforming (BF) as long as channel knowledge is not outdated and switches to space frequency block coding (SFBC) afterwards. Approximate BER expressions for BF and SFBC that are functions of SNR, modulation order, Doppler frequency and initial channel knowledge are derived to be used as a decision metric for mode allocation throughout the frame. Second method adapts rate and transmission mode across symbols in a frame by considering that average channel power is monotonically decreasing as a function of time within the frame when multiuser diversity is exploited. First method performs as good as the better of BF and SFBC over all SNR values. Second method provides additional performance gain over the first method due to more efficient use of better channel conditions.
\end{abstract}

Index Terms-MIMO, OFDMA, time varying channel, nonuniform channel knowledge, statistical bit loading, transmission mode allocation.

\section{INTRODUCTION}

$\mathbf{N}$ EXT generation wireless communication systems promise higher data rates and more reliable communication in diverse propagation environments. An important aspect of designing a good communication system relies on efficient utilization of available diversity in the system. Orthogonal frequency division multiple access (OFDMA) modems employing multiple antennas allow a flexible system design in which frequency and spatial diversity of the channel can be exploited. When the channel is frequency-selective, frequency diversity can be extracted by assigning mobile users to their best channel out of finite number of subchannels which can also be termed as multiuser diversity. Multiple antennas can be used in a variety of ways to improve the link quality. Beamforming (BF) and precoding techniques are used to provide array gain, when the channel knowledge is available at the transmitter. Alternatively, space-frequency coding schemes can be used to exploit

Paper approved by N. C. Beaulieu, the Editor for Wireless Communication Theory of the IEEE Communications Society. Manuscript received October 30, 2009; revised November 30, 2010.

B. Kecicioglu and H. Minn are with the Department of Electrical Engineering, The University of Texas at Dallas, Richardson, TX, 75083, USA (e-mail: \{bxk022000, Hlaing.Minn\}@utdallas.edu).

C.-C. Chong was with DOCOMO USA Labs, Palo Alto, CA, 94304, USA (e-mail: chia-chin.chong@ieee.org).

Digital Object Identifier 10.1109/TCOMM.2011.062111.090659 spatial diversity in the channel without requiring any channel information at the transmitter.

The channel information can be acquired either through feedback from the receiver both in frequency division duplex (FDD) and time division duplex (TDD) systems, or by measuring the uplink channel in TDD systems ${ }^{1}$. In both cases, channel knowledge at the transmitter is not perfect. It is essential to consider channel knowledge imperfections at the transmitter due to estimation errors, quantization errors, feedback delays and channel time variations. In recent years, researchers have focused on how to optimize multiple antenna transmission with imperfect channel knowledge at the transmitter. Authors in [1]-[4] studied optimum transmission strategy when partial channel knowledge is available at the transmitter. An alternative approach is to combine space diversity schemes with beamforming to provide a robust transmission based on the quality of the channel knowledge [5]-[9]. All of these works rely on the quasi-static fading assumption in which the channel is considered fixed throughout the frame. Hence, these analyses are based on a constant channel imperfection. However, this assumption may not be valid for environments with a relatively large ratio of Doppler spread to frame rate. As International Telecommunications Union (ITU)'s recommendation for further enhancements to next generation wireless systems includes mobility support up to $350 \mathrm{~km} / \mathrm{h}$ mobile speed [18], many scenarios in LTE and Wimax systems can be considered under this cathegory [19], [20]. Table I shows ratios of the Doppler spread to the symbol rate and to the frame rate with respect to different mobile speeds for LTE and Wimax systems. It should be noted that the frame definition in this paper is the time duration between two consecutive channel state information acquisitions. Since LTE frame structure may consist of multiple uplink (UL) and downlink (DL) subframes, the time duration between two channel knowledge acquisitions can be a subframe for FDD system and two subframes for TDD system. In Wimax system, channel knowledge can be updated once in each frame. In Section V, we show effects of these values on the system performance.

In this paper, we consider a fast fading channel model in which the channel can change during the frame. Thus, the amount of correlation between the actual channel and initial channel knowledge changes from symbol to symbol. We propose two simple MIMO switching methods in which the transmitter chooses between the space-frequency block coding (SFBC) and BF to minimize BER. Given that the channel quality may degrade through the frame for high mobile speeds,

\footnotetext{
${ }^{1}$ The transceiver circuitry needs to be designed properly to provide reciprocal channels.
} 
different transmission modes are allowed in a single frame. In the first method, MIMO transmission modes are allocated for a given frame. Transmitter calculates the switching point inside the frame based on the initial channel knowledge and time-domain correlation properties of the channel. Although the channel changes across the frame, the transmitter does not need to know instantaneous channel knowledge. This mode allocation algorithm requires knowledge of BER performance of BF and SFBC as a function of system parameters at the transmitter. For this purpose we derived analytical BER expressions for diversity and beamforming methods conditioned on the initial channel knowledge. Given the initial channel knowledge, average SNR, data rate and Doppler frequency, the transmitter allocates transmission modes inside the frame. The initial transmission mode and the switching point information is included in the frame header which enables the receiver to decode the frame.

In the second method, both multiple antenna transmission mode and data rate are varied across symbols in a given frame by considering nonuniform channel statistics induced by multiuser and frequency diversity exploitation. Numerical results show that the first method outperforms individual $\mathrm{BF}$ and SFBC transmissions for diverse mobile speeds. The second method performs better than the first method when frequency or spatial diversity of the system increases. We also consider the adaptation that is based on the BER performance averaged over channel statistics. In this approach adaptation is performed less frequently since the adaptation criterion is related to long term channel statistics.

The rest of the paper is organized as follows. In Section II, our system model is presented. The first method is explained and BER performance equations to be used as decision metrics are provided in Section III. Section IV gives description of the second method and presents derivation of average BER expressions to be used in the mode allocation and bit loading algorithm. Numerical results are presented in Section V and the paper is concluded in Section VI.

\section{SySTEM Model}

We consider downlink (DL) of a wireless network employing OFDMA for multiuser access. The transmitter has $n_{t}$ antennas whereas each mobile user has $n_{r}$ receive antennas. $Q$ subchannels are formed by grouping consecutive subcarriers over which the channel is highly correlated. Therefore each subchannel can be represented by a single channel matrix. The low-pass equivalent of the received signal of user $k$ on subchannel $q$ at symbol time $n$ is given by

$$
\mathbf{y}^{k}(n)=\mathbf{H}_{q, n}^{k} \mathbf{x}_{q}^{k}(n)+\mathbf{w}_{q}^{k}(n)
$$

where $\mathbf{w}_{q}^{k}(n)$ is the $n_{r} \times 1$ noise vector. We assume that noise is circularly symmetric complex Gaussian with zero mean and unit variance and it is uncorrelated across antennas. $\mathbf{H}_{q, n}^{k}$ is the $n_{r} \times n_{t}$ matrix containing channel coefficients of user $k$ on subchannel $q$. In this paper, it is assumed that the channels are statistically independent and identically distributed (iid) between different users. Each channel coefficient is distributed as circularly symmetric complex Gaussian with zero mean and unit variance. $\mathbf{x}_{q}^{k}$ is the transmitted signal vector for user $k$ on

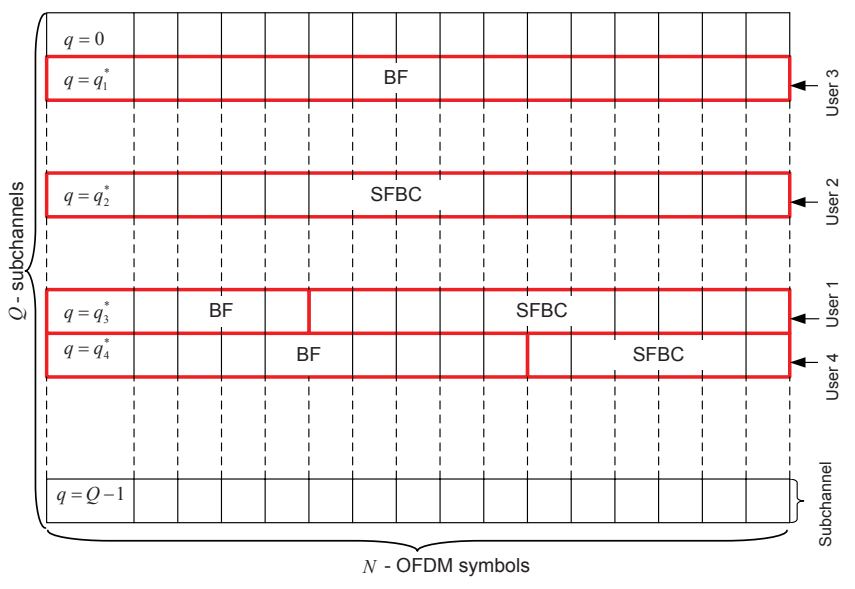

Fig. 1. Frame structure of the proposed system.

subchannel $q$ with average power $\mathrm{E}\left[\left\|\mathbf{x}_{q}^{k}(n)\right\|^{2}\right]=\eta$, therefore $\eta$ is the average SNR per receive antenna.

\section{A. Channel Model}

In the present study, we consider a fast-fading channel where the channel varies during a frame. Therefore the quasistatic fading assumption is not valid at a frame level but it is still assumed that the channel stays highly correlated during each OFDM symbol. This fast fading channel effect can be observed for high Doppler frequencies or for long frame durations. For this scenario, the channel knowledge available at the beginning of the frame becomes degraded towards the end of the frame and outdated channel matrix at symbol time $n, \mathbf{H}_{q, n}^{k}$, is given by [15]

$$
\mathbf{H}_{q, n}^{k}=\rho_{n} \mathbf{H}_{q,-\tau}^{k}+\sqrt{1-\rho_{n}^{2}} \mathbf{H}_{e, q, n}^{k}
$$

where $\mathbf{H}_{q,-\tau}^{k}$ is the channel knowledge at the beginning of the frame and $\mathbf{H}_{e, q, n}^{k}$ is the perturbation term due to decorrelation effect between the initial channel knowledge and the actual channel. Here, $\tau$ is a positive integer representing channel feedback delay in number of symbols. Note that $\mathbf{H}_{q,-\tau}^{k}=\mathbf{H}_{q, 0}^{k}$ when there is no feedback delay. $\rho_{n}$ is the correlation coefficient between the initial channel knowledge $\mathbf{H}_{q,-\tau}^{k}$ and the current channel realization $\mathbf{H}_{q, n}^{k}$ at symbol time $n$. Although channel is changing during a frame, the receiver can estimate the channel with pilot symbols spread over the frame in time-frequency grid. Thus, in this work we assume that the receiver has perfect channel knowledge for all decoding purposes. The channel is correlated in spatial domain with covariance matrix $E\left[\operatorname{vec}\left(\mathbf{H}_{q}^{k}\right) \operatorname{vec}\left(\mathbf{H}_{q}^{k}\right)^{H}\right]=\mathbf{R}$. We use so called Kronecker model in our analysis. In this model, channel correlation at the transmitter and receiver sides are assumed to be separate and covariance matrix is given by $\mathbf{R}=\mathbf{R}_{\mathrm{tx}} \otimes \mathbf{R}_{\mathrm{rx}}$, where $\mathbf{R}_{\mathrm{tx}}$ and $\mathbf{R}_{\mathrm{rx}}$ are covariance matrices at the transmitter and receiver sides respectively and the symbol $\otimes$ represents Kronecker product operator. We define eigen decompositions of covariance matrices as $\mathbf{R}=\mathbf{U} \boldsymbol{\Sigma} \mathbf{U}^{H}, \mathbf{R}_{\mathrm{tx}}=\mathbf{U}_{\mathrm{tx}} \boldsymbol{\Sigma}_{\mathrm{tx}} \mathbf{U}_{\mathrm{tx}}^{H}$ and $\mathbf{R}_{\mathrm{rx}}=\mathbf{U}_{\mathrm{rx}} \boldsymbol{\Sigma}_{\mathrm{rx}} \mathbf{U}_{\mathrm{rx}}^{H}$, where $\boldsymbol{\Sigma}_{\mathrm{tx}}$ and $\boldsymbol{\Sigma}_{\mathrm{rx}}$ are diagonal matrices given as $\boldsymbol{\Sigma}_{\mathrm{tx}}=$ 
TABLE I

THE DEGREE OF CHANNEL TIME VARIATIONS IN PRACTICAL WIRELESS SYSTEMS

\begin{tabular}{|c|c|c|c|c|c|c|c|c|}
\hline & $v($ & 50 & 100 & 150 & 200 & 250 & 300 & 350 \\
\hline \multirow{2}{*}{$\begin{array}{c}\text { Wimax } \\
\left(f_{c}=2.4 \mathrm{GHz}, T_{s}=108.3 \mu \mathrm{s}, T_{f}=5.2 \mathrm{~ms}\right)\end{array}$} & & 0.012 & 0.0241 & 0.0361 & 0.0481 & 0.0602 & 0.0722 & 0.0842 \\
\hline & $f_{d} T_{f}$ & & & & & & & 4.0432 \\
\hline \multirow{2}{*}{$\begin{array}{c}\text { LTE } \\
\left(f_{c}=2 \mathrm{GHz}, T_{s}=71.4 \mu \mathrm{s}, T_{f}=1 \mathrm{~ms}\right)\end{array}$} & $f_{d} T_{s}$ & 0.4 & & & & & 0.0397 & 0.0463 \\
\hline & $f_{d} T_{f}$ & 0.0926 & 0.1852 & 0.2778 & 0.3704 & 0.4630 & 0.5556 & 0.6481 \\
\hline
\end{tabular}

$\operatorname{diag}\left(\nu_{1}, \nu_{2}, \ldots, \nu_{n_{t}}\right)$ and $\boldsymbol{\Sigma}_{\mathrm{rx}}=\operatorname{diag}\left(\mu_{1}, \mu_{2}, \ldots, \mu_{n_{r}}\right)$. It is known that $\boldsymbol{\Sigma}=\boldsymbol{\Sigma}_{\mathrm{tx}} \otimes \boldsymbol{\Sigma}_{\mathrm{rx}}$. Here, it should be noted that we do not assume full rank covariance matrices in the following analysis. Rank of the covariance matrices depends on scattering properties of the channel.

\section{B. Channel Assignment}

OFDMA systems can harness frequency and multiuser diversity of the propagation environment by adaptive channel assignment. As shown in Figure 1, available spectrum is divided into subchannels consisting of consecutive subcarriers and mobile users are assigned to different subchannels depending on their channel conditions. BS obtains channel information at the beginning of the frame and performs channel assignment and transmission mode selection for the mobile users for each frame.

BS has channel knowledge $\mathbf{H}_{q,-\tau}^{k}$ of all users at the beginning of the frame for $q=0, \ldots, Q-1$. Based on this initial knowledge, channels are assigned to users. However, channel decorrelates (with respect to the initial channel knowledge) at symbol time $n$ according to the model in (2). In the model, $\rho_{n}$ is an arbitrary correlation coefficient determined by timeselectivity of the channel. It is clear that benefits of adaptive channel assignment diminish with time as the initial channel knowledge $\mathbf{H}_{q,-\tau}^{k}$ becomes outdated. Therefore, frequency and multiuser diversity can be utilized for a fraction of the frame in the beginning and this fraction of time depends on Doppler frequency spread.

In practical systems, channel assignment method may depend on the quality-of-service $(\mathrm{QoS})$ requirements and fairness constraints. In our analysis we assume that a given mobile user is always assigned to its best channel. Therefore, we drop user index $k$ and channel index $q$ when it is clear from the context.

BS computes the best subchannel for each transmission mode and then selects the best initial transmission mode and corresponding best subchannel. The selected subchannel occupies the same subcarriers throughout the frame but MIMO transmission mode may change. Examples of possible channel and transmission mode allocations is shown in Figure 1. Since the channel with the largest eigenvalue gives better performance for single mode beamforming, the transmitter selects the channel with the largest maximum eigenvalue. Therefore, the selected channel index $q^{*}$ is given by

$$
q_{\mathrm{bf}}^{*}=\underset{q}{\arg \max } \lambda_{\max , q}
$$

where $\lambda_{\max , q}$ is the largest eigenvalue of the matrix $\mathbf{H}_{q,-\tau}^{H} \mathbf{H}_{q,-\tau}$. For the SFBC, SNR maximizing channel is the one with the largest Frobenius norm. Therefore, the channel assignment criterion for SFBC is

$$
q_{\mathrm{sfbc}}^{*}=\underset{q}{\arg \max }\left\|\mathbf{H}_{q,-\tau}\right\|_{F}^{2}
$$

where $\|\cdot\|_{F}$ denotes Frobenius norm operator. From now on, we denote $g_{\mathrm{bf}}=\lambda_{\max , q_{\mathrm{bf}}^{*}}$ and $g_{\mathrm{sfbc}}=\left\|\mathbf{H}_{q_{\mathrm{sfbc}}^{*},-\tau}\right\|_{F}^{2}$. Note that for multiple-input single-output (MISO) system, channel selection criteria is the same for both BF and SFBC. Specifically, the best channel is the one with largest Frobenius norm.

\section{Method I: Mimo Mode Allocation Based on INITIAL CHANNEL KNOWLEDGE}

In the first method, MIMO transmission modes are allocated throughout the frame depending on the channel knowledge at the beginning of the frame, spatial covariance matrix, average SNR, Doppler frequency of mobile user and data rate. We consider single mode BF and orthogonal SFBC as alternative transmission methods. Having the initial channel knowledge for all subchannels, BS chooses the best subchannel. Conditioned on the selected channel knowledge and correlation coefficients $\rho_{n}$ at the $n$th symbol time, BS computes average BER for every symbol in the frame and allocates transmission modes based on the minimum average BER criterion.

In the following, we derive average BER conditioned on the initial channel knowledge for BF and SFBC for each symbol in the frame. These BER expressions are used to select between two transmission modes for each symbol time. In the analysis, we only consider $M$-ary quadrature amplitude modulated ( $M$ QAM) signal and we use the approximate BER expression

$$
P_{b}(\gamma) \approx 0.2 e^{-\frac{3}{2(M-1)} \gamma}
$$

from [13], where $\gamma$ is the SNR per symbol over each subcarrier. After obtaining initial channel knowledge, average BERs of BF and SFBC are calculated and MIMO transmission mode is selected for each symbol to minimize BER for a fixed rate and fixed power transmission. The transmission modes are also known to the mobile user by means of control information from BS or by performing the same selection algorithm at the receiver. Using the approximate BER expression in (5), average BER performance can be obtained as

$$
P_{b} \approx 0.2 \int_{0}^{\infty} e^{-\frac{3}{2(M-1)} \gamma} f_{\gamma}(\gamma) d \gamma=0.2 \phi_{\gamma}\left(-\frac{3}{2(M-1)}\right)
$$

where $\phi_{\gamma}(s)$ is the moment generating function (MGF) of $\gamma$ [7].

\section{A. BER of BF with Initial Channel Knowledge}

Channel knowledge at the transmitter can be used to provide array gain by transmitting in the direction of the dominant eigenvector of the channel matrix. If the channel knowledge is not perfect, performance degrades due to mismatch between eigenvectors of the available channel knowledge $\mathbf{H}_{-\tau}$ and the actual channel $\mathbf{H}_{n}$. 
Let singular value decomposition (SVD) of $\mathbf{H}_{n}$ be given by $\mathbf{H}_{n}=\mathbf{U}_{n} \boldsymbol{\Sigma}_{n} \mathbf{V}_{n}^{H}$, where $\mathbf{U}_{n}=\left[\mathbf{u}_{1, n}, \ldots, \mathbf{u}_{d, n}\right]$ and $\mathbf{V}_{n}=\left[\mathbf{v}_{1, n}, \ldots, \mathbf{v}_{d, n}\right]$ are unitary matrices composed of left and right singular vectors of the channel matrix $\mathbf{H}_{n}$ and $d \leq \min \left(n_{r}, n_{t}\right)$ is the rank of the channel. $\boldsymbol{\Sigma}_{n}=$ $\operatorname{diag}\left(\left[\sqrt{\lambda_{1, n}}, \ldots, \sqrt{\lambda_{d, n}}\right]\right)$ is a diagonal matrix having ordered singular values on its diagonal where $\lambda_{1, n}, \ldots, \lambda_{d, n}$ are eigenvalues of $\mathbf{H}_{n}^{H} \mathbf{H}_{n}$. In the single mode beamforming, the transmitter performs beamforming in the direction of the largest eigenvalue of the matrix $\mathbf{H}_{n}^{H} \mathbf{H}_{n}$ in order to maximize the received SNR using the dominant eigenvector $\mathbf{v}_{1, n}$. A similar treatment for multimode beamforming can be found in [16]. In the current system, the transmitter has channel knowledge at the beginning of the frame as $\mathbf{H}_{-\tau}$. Therefore the transmitter modulates the signal using the vector $\mathbf{v}_{1,-\tau}$ instead of $\mathbf{v}_{1, n}$. The received signal vector at symbol $n$ is then given by

$$
\mathbf{y}_{n}=\mathbf{H}_{n} \mathbf{v}_{1,-\tau} x_{n}+\mathbf{w}_{n}
$$

where $x_{n}$ is the transmitted symbol and $\mathbf{w}_{n}$ is the $n_{r} \times 1$ noise vector at the receiver. Average power of the transmitted signal is $E\left[\left|x_{n}\right|^{2}\right]=\eta$ and $\mathbf{w}_{n}$ is distributed as $\mathcal{C N}\left(0, \mathbf{I}_{n_{r}}\right)$. Note that the receiver can reliably estimate the channel vector throughout the frame. Therefore, maximal-ratio-combining (MRC) can be employed at the receiver with combining weight vector $\mathbf{g}_{n}=\mathbf{H}_{n} \mathbf{v}_{1,-\tau} /\left\|\mathbf{H}_{n} \mathbf{v}_{1,-\tau}\right\|$ to obtain

$$
r_{n}=\mathbf{g}_{n}^{H} \mathbf{y}_{n}=\left\|\mathbf{H}_{n} \mathbf{v}_{1,-\tau}\right\| x_{n}+\tilde{w}_{n}
$$

where $\tilde{w}_{n}$ is the noise after combining. Note that the received SNR is given by $\gamma_{n}=\eta\left\|\mathbf{H}_{n} \mathbf{v}_{1,-\tau}\right\|^{2}$. According to the channel model in (2), the random vector $\mathbf{z}_{n}=\sqrt{\eta} \mathbf{H}_{n} \mathbf{v}_{1,-\tau}$ is given by

$$
\begin{aligned}
\mathbf{z}_{n} & =\sqrt{\eta}\left(\rho_{n} \mathbf{H}_{-\tau}+\sqrt{1-\rho_{n}^{2}} \mathbf{H}_{e, n}\right) \mathbf{v}_{1,-\tau} \\
& =\sqrt{\eta}\left(\rho_{n} \sqrt{g_{\mathrm{bf}}} \mathbf{u}_{1,-\tau}+\sqrt{1-\rho_{n}^{2}} \mathbf{h}_{e, n}\right)
\end{aligned}
$$

where $\mathbf{h}_{e, n}=\mathbf{H}_{e, n} \mathbf{v}_{1,-\tau}$ and $\gamma_{n}=\left\|\mathbf{z}_{n}\right\|^{2}$. Note that SNR would be $\tilde{\gamma}=\eta g_{\mathrm{bf}}$ if the channel was not fast fading and there was no feedback delay. Conditioned on the initial channel knowledge $\mathbf{H}_{-\tau}, \mathbf{z}_{n}$ is a complex Gaussian distributed random vector with mean $\sqrt{\eta g_{\mathrm{bf}}} \rho_{n} \mathbf{u}_{1,-\tau}$ and covariance matrix $\eta\left(1-\rho_{n}^{2}\right)\left(\mathbf{v}_{1,-\tau}^{H} \mathbf{R}_{\mathrm{tx}} \mathbf{v}_{1,-\tau}\right) \mathbf{R}_{\mathrm{rx}}$. As pointed out in (6), finding MGF of $\gamma_{n}$ is sufficient to obtain approximate BER metric. Therefore we need to find MGF of $\gamma_{n}=\left\|\mathbf{z}_{n}\right\|^{2}$, which is already given in [8] as (10) on top of the next page. This expression can be further simplified to obtain (11). Here, $\tilde{u}_{1,-\tau}(m)$ is the $m$ th element of the channel vector $\tilde{\mathbf{u}}_{1,-\tau}$ that is obtained by $\tilde{\mathbf{u}}_{1,-\tau}=\mathbf{U}_{\mathrm{rx}}^{H} \mathbf{u}_{1,-\tau}$. Finally, the average BER expression at symbol $n$ conditioned on the initial channel knowledge $\mathbf{H}_{-\tau}$ as shown in (12). In (12), $M_{n}$ is the $M$-QAM alphabet size used for $n$th symbol. Using this result, average BER conditioned on the initial channel knowledge $\mathbf{H}_{-\tau}$ is given by

$$
P_{b}^{\mathrm{bf}}(\tilde{\gamma}) \approx \frac{1}{N} \sum_{n=0}^{N-1} P_{b}^{\mathrm{bf}}\left(n, M_{n}, \tilde{\gamma}\right),
$$

where $N$ is the number of OFDM symbols in a frame.

\section{B. BER of SFBC Conditioned on Initial Channel Knowledge}

SFBC is an effective way of exploiting spatial diversity of the channel when channel knowledge is not available at the transmitter. In SFBC, a block of $m$ modulated symbols are coded across $n_{f}$ subcarriers and coded vectors are simultaneously transmitted from $n_{t}$ antennas. Rate of such a SFBC is $R=m / n_{f}$. In this paper, we optimize transmission mode to minimize BER for a fixed rate and fixed power transmission. If the rate of SFBC is $R<1$, then modulation order of SFBC is increased to satisfy constant rate requirement. Considering the system model in (1), we can write

$$
\mathbf{y}_{n}=\mathbf{H}_{n} \mathbf{x}_{n}+\mathbf{w}_{n}
$$

where $\mathbf{x}_{n}$ is $n_{t} \times 1$ transmitted signal vector generated according to a given SFBC matrix. In this work, we consider orthogonal SFBC designs for their very low decoding complexity. Assuming that the channel is highly correlated across consecutive subcarriers, receiver can decode symbols with linear complexity. Symbols from each antenna are normalized by $1 / \sqrt{n_{t}}$ to satisfy constant power requirement, i.e., $E\left[\left\|\mathbf{x}_{n}\right\|^{2}\right]=\eta$. Thus, the received SNR is given by $\gamma_{n}=a \frac{\eta}{n_{t}}\left\|\mathbf{H}_{n}\right\|_{F}^{2}$ [14], where $a$ is a parameter that is a function of SFBC code. $a=1$ for Alamouti code and $a=2$ for rate $1 / 2$ code designed for 4 transmit antennas [14]. Here, we define SNR $\tilde{\gamma}$ in the absence of channel delay and fast fading as $\tilde{\gamma}=a \frac{\eta}{n_{t}} g_{\mathrm{sfbc}}$. By stacking columns of $\mathbf{H}_{n}$ in a vector, we define $\overline{\mathbf{h}}_{n} \triangleq \operatorname{vec}\left(\mathbf{H}_{n}\right)$. Therefore, $\mathrm{SNR}$ at symbol time $n$ is given by $\gamma_{n}=a \frac{\eta}{n_{t}}\left\|\overline{\mathbf{h}}_{n}\right\|^{2}$. From the channel model in (2), $\overline{\mathbf{h}}_{n}$ conditioned on the initial channel knowledge $\overline{\mathbf{h}}_{-\tau}$ is distributed as $\mathcal{C N}\left(\rho_{n} \overline{\mathbf{h}}_{-\tau},\left(1-\rho_{n}^{2}\right) \mathbf{R}\right)$, where $\mathbf{R}=\mathbf{R}_{\mathrm{tx}} \otimes \mathbf{R}_{\mathrm{rx}}$ as defined in Section II.A. Following similar steps to arrive at (12), the average BER performance of SFBC for $M$-QAM at symbol $n$ can be written as shown on top of the next page in (15). In (15), $\tilde{\mathbf{H}}_{-\tau}=\operatorname{unvec}\left(\mathbf{U}^{H} \overline{\mathbf{h}}_{-\tau} /\left\|\overline{\mathbf{h}}_{-\tau}\right\|\right)$. Here "unvec" is the inverse of "vec" operator. Again, average BER for a given average $\mathrm{SNR} \eta$ is given by

$$
P_{b}^{\mathrm{sfbc}}(\tilde{\gamma}) \approx \frac{1}{N} \sum_{n=0}^{N-1} P_{b}^{\mathrm{sfbc}}\left(n, M_{n}, \tilde{\gamma}\right) .
$$

In this work, we consider a fast fading channel for which quasi-static assumption does not hold. BS obtains channel information either through feedback for non-reciprocal channels (FDD systems) or via uplink measurement for reciprocal channels (TDD systems). Therefore, both BS and mobile user have channel information $\mathbf{H}_{-\tau}$ at the beginning of the frame. On the other hand, receiver has perfect channel information as well. We also assume that both sides have knowledge of mobile user speed and channel feedback delay. With this information, average BER for BF and SFBC can be calculated at the BS and mobile user by using (12) and (15). Then, MIMO transmission mode is assigned based on

$$
m^{*}(n)=\underset{m \in\{\mathrm{bf}, \mathrm{sfbc}\}}{\arg \min } P_{b}^{m}\left(n, M_{n}, \tilde{\gamma}\right),
$$

where $m^{*}(n)$ is the transmission mode index on symbol $n$. Alternatively, mobile user can be informed about initial transmission mode and mode switching point via control information in the packet header. By doing this, complexity 


$$
\begin{gathered}
\phi_{\gamma_{n}}(s)=\frac{\exp \left(s \rho_{n}^{2} \tilde{\gamma} \mathbf{u}_{1,-\tau}^{H}\left(\mathbf{I}-s \eta\left(1-\rho_{n}^{2}\right)\left(\mathbf{v}_{1,-\tau}^{H} \mathbf{R}_{\mathrm{tx}} \mathbf{v}_{1,-\tau}\right) \mathbf{R}_{\mathrm{rx}}\right)^{-1} \mathbf{u}_{1,-\tau}\right)}{\left|\mathbf{I}-s \eta\left(1-\rho_{n}^{2}\right)\left(\mathbf{v}_{1,-\tau}^{H} \mathbf{R}_{\mathrm{tx}} \mathbf{v}_{1,-\tau}\right) \mathbf{R}_{\mathrm{rx}}\right|}(10) \\
\phi_{\gamma_{n}}(s)=\prod_{m=1}^{n_{r}}\left(1-s \eta\left(1-\rho_{n}^{2}\right)\left(\mathbf{v}_{1,-\tau}^{H} \mathbf{R}_{\mathrm{tx}} \mathbf{v}_{1,-\tau}\right) \mu_{m}\right)^{-1} \exp \left(s \rho_{n}^{2} \tilde{\gamma} \sum_{m=1}^{n_{r}} \frac{\left|\tilde{u}_{1,-\tau}(m)\right|^{2}}{1-s \eta\left(1-\rho_{n}^{2}\right)\left(\mathbf{v}_{1,-\tau}^{H} \mathbf{R}_{\mathrm{tx}} \mathbf{v}_{1,-\tau}\right) \mu_{m}}\right) \\
P_{b}^{\mathrm{bf}}\left(n, M_{n}, \tilde{\gamma}\right) \approx 0.2 \prod_{m=1}^{n_{r}}\left(1+\frac{1.5 \eta\left(1-\rho_{n}^{2}\right)\left(\mathbf{v}_{1,-\tau}^{H} \mathbf{R}_{\mathrm{tx}} \mathbf{v}_{1,-\tau}\right) \mu_{m}}{M_{n}-1}\right)^{-1} \exp \left(\frac{-1.5 \rho_{n}^{2} \tilde{\gamma}}{M_{n}-1} \sum_{m=1}^{n_{r}} \frac{\left|\tilde{u}_{1,-\tau}(m)\right|^{2}}{1+\frac{1.5 \eta\left(1-\rho_{n}^{2}\right)\left(\mathbf{v}_{1,-\tau}^{H} \mathbf{R}_{\mathrm{tx}} \mathbf{v}_{1,-\tau}\right) \mu_{m}}{M_{n}-1}}\right) \\
P_{b}^{\mathrm{sfbc}}\left(n, M_{n}, \tilde{\gamma}\right) \approx 0.2 \prod_{k=1}^{n_{m}} \prod_{m=1}^{n_{r}}\left(1+\frac{1.5 a \eta\left(1-\rho_{n}^{2}\right) \mu_{m} \nu_{k}}{n_{t}\left(M_{n}-1\right)}\right)^{-1} \exp \left(-\frac{1.5 \rho_{n}^{2} \tilde{\gamma}}{\left(M_{n}-1\right)} \sum_{k=1}^{n_{t}} \sum_{m=1}^{n_{r}} \frac{\left|\tilde{H}_{-\tau}\left(k, m_{m}\right)\right|^{2}}{1+\frac{1.5 a \eta\left(1-\rho_{n}^{2}\right) \mu_{m} \nu_{k}}{\left(M_{n}-1\right) n_{t}}}\right) \\
(15)
\end{gathered}
$$

can be reduced in the mobile user side and a possible mismatch between the BS and mobile user about the location of the switching point can be avoided.

\section{Method II: MIMO TRANSMission MODE Allocation AND Bit LoAding}

In the second method, both rate and transmission mode is adapted across symbols to minimize average BER. When the channel knowledge is exploited by BF and channel selection, average channel power is higher at the beginning of the frame. As the channel decorralates, CSI becomes outdated and average channel power decreases throughout the frame. It is well known that adaptive bit loading improves the performance when channel quality varies. The proposed bit loading algorithm makes use of the better channel conditions at the beginning of frame by transmitting with higher data rate at the beginning. First, MIMO transmission modes are allocated based on the criterion given in (17). After this decision, bit loading algorithm is performed to assign modulation orders to each symbol. The corresponding optimization problem can be expressed as

$$
\begin{aligned}
\left(M_{1}^{*}, M_{2}^{*}, \ldots, M_{N}^{*}\right) & =\underset{\left(M_{1}, M_{2}, \ldots, M_{N}\right)}{\arg \min } \sum_{n=1}^{N} \mathrm{P}_{b}^{m^{*}(n)}\left(n, M_{n}, \tilde{\gamma}\right) \\
2^{R} & =\sum_{n=1}^{N} M_{n} \\
M_{n} & \leq 2^{r_{\max }} \text { for } n \in\{1,2, \ldots, N\}
\end{aligned}
$$

where $M_{n}$ is the modulation order on the $n$th symbol time and $R$ is the rate constraint in number of bits per frame for a given mobile user and $r_{\max }$ is the instantaneous rate constraint in number of bits. Solution to this optimization problem can be found by a simple iterative algorithm. The iterative algorithm adds certain number of bits to the frame in each step such that it loads bits to the symbol causing minimum increase in BER. Note that the number of bits to be loaded in each step depends on the candidate modulation orders $M_{n}$. The algorithm loads $r$ bits in each step if $\log _{2}\left(M_{n}\right)$ increases with minimum step size of $r$ bits. If this is not feasible in any step, the number of bits to be loaded is increased to $2 r$ bits in the next iteration. Therefore, $r$ bits are loaded in each iteration and this algorithm terminates after $R / r$ steps. If the $r_{\max }$ is reached for any symbol $n$, then modulation order of that symbol is finalized as $M_{n}=2^{r_{\max }}$. In our system, candidate modulation orders are 4-QAM, 16-QAM, 64-QAM and 'no transmission'. Hence $M_{n}$ increases in steps of $r=2$ bits and $r_{\max }=6$ bits.

Adapting rate together with MIMO transmission mode provides performance gain at the cost of increased complexity. The complexity of this system can be reduced if it is implemented based on unconditional BER metric instead of conditional BER expressions in (12) and (15). This requires BER expression averaged over the initial channel statistics $f_{\tilde{\gamma}}(\tilde{\gamma})$. Therefore, we need to evaluate

$$
\mathrm{P}_{b}^{m^{*}(n)}\left(n, M_{n}\right)=\int_{0}^{\infty} \mathrm{P}_{b}^{m^{*}(n)}\left(n, M_{n}, \tilde{\gamma}\right) f_{\tilde{\gamma}}(\tilde{\gamma}) d \tilde{\gamma}
$$

for all $n=1,2, \ldots, N$. For this purpose we need to know $f_{\tilde{\gamma}}(\tilde{\gamma})$. Due to the subchannel selection, $f_{\tilde{\gamma}}(\tilde{\gamma})$ is order statistics of SNR distribution on each subchannel in the frequency domain. Denoting PDF of SNR on each subchannel as $f_{\gamma_{s}}\left(\gamma_{s}\right)$ and cumulative distribution function (CDF) as $F_{\gamma_{s}}\left(\gamma_{s}\right)$, we can write $\mathrm{CDF}$ of $\tilde{\gamma}$ as $F_{\tilde{\gamma}}(\tilde{\gamma})=\left(F_{\gamma_{s}}\left(\gamma_{s}\right)\right)^{d_{f}}$, where $d_{f}$ is the diversity order due to frequency and multiuser diversity exploitation. In our system, we consider a given user that is assigned to its best subchannel. In this case, frequency diversity depends on frequency selectivity of the channel. If time domain channel coefficients are assumed to be identically distributed with a uniform delay profile and the number of time domain channel taps is $N_{\text {tap }}$, then channel selection from $Q$ subchannels is approximately equivalent to selection among $N_{\text {tap }}$ independent subchannels. Therefore, $d_{f}$ can be approximated as $d_{f} \approx N_{\text {tap. }}$. In practical channels, time domain channel coefficients have decaying power delay profile but still number of significant taps can give a good estimate of frequency diversity order.

Obtaining closed form expression for $f_{\tilde{\gamma}}(\tilde{\gamma})$ in its most general form is not analytically tractable. However, we provide closed form BER expressions for MISO systems with uncorrelated antennas when $n_{t}=2,4$ in the Appendix. 


\section{Simulations And Results}

We tested our algorithm with two different channel models. The first is based on the first order auto-regressive (AR1) model in [10]. Channel correlation coefficient $\alpha$ between the initial channel knowledge and the channel knowledge after first symbol duration is calculated from $\alpha=J_{0}\left(2 \pi f_{d} T_{s}\right)$, where $J_{0}($.$) is the zeroth order Bessel function, T_{s}$ is the symbol duration and $f_{d}$ is the Doppler frequency $\left(f_{d}=\frac{v f_{c}}{c}\right.$, $v$ is the speed of the mobile user with respect to BS and $f_{c}$ is the carrier frequency). According to the AR1 model, correlation coefficients during symbol time $n$ is found from $\rho_{n}=\alpha^{n+\tau}$ where $n=0,1, \ldots, N-1$. Channel coefficients are uncorrelated across antennas. Rayleigh fading is considered with uniform power delay profile. Although it is very simple, this model provides performance limits of the system for a predetermined frequency and time selectivity. The second set of simulations are performed with a realistic MIMO channel model that is developed for WINNER project [17]. The simulations are conducted for rural macrocell environment since it supports mobile speeds of up to $200 \mathrm{~km} / \mathrm{h}$. LTE baseband system parameters are used with a center frequency of $2 \mathrm{GHz}$.

Discrete-Fourier-Transform (DFT) size is 1024, where out of 1024 subcarriers, 576 of them are used as in LTE. Used subcarriers are grouped into 24 subchannels consisting of 24 consecutive subcarriers. The receiver has perfect channel knowledge throughout the frame and the transmitter obtains channel knowledge at the beginning of the frame with or without feedback delay. When performing $\mathrm{BF}$ in a subchannel, BF vectors are computed for each subcarrier. When implementing SFBC, full rate Alamouti code is used for $n_{t}=2$, however rate $1 / 2$ code is used if $n_{t}=4$. Therefore, the modulation order of $\mathrm{BF}, M_{\mathrm{bf}}$, is equal to the modulation order of SFBC, $M_{\mathrm{sfbc}}$, when $n_{t}=2$, whereas $M_{\mathrm{sfbc}}=M_{\mathrm{bf}}^{2}$ when $n_{t}=4$ to maintain the same data rate.

\section{A. Proposed Method I: MIMO Transmission Mode Allocation Based on the Initial Channel Knowledge}

First, we show performance of channel-adaptive transmission method described in Section III. In this method, MIMO transmission modes are allocated based on instantaneous initial channel knowledge, Doppler frequency, average SNR and modulation order. In Figure 2, the performance is shown for $2 \times 2$ antenna configuration for AR1 channel model. The transmission method I performs as good as or slightly better than the best of BF and SFBC transmissions over all SNR range. In this figure, normalized Doppler frequency $f_{d} T_{s}$ is appropriately selected to show a crossover point between average BER of SFBC and BF, which occurs at a particular average SNR value $\eta_{\mathrm{th}}$. Performance of the proposed method for the WINNER channel model is presented in Figure 3 for $2 \times 2$ antenna configuration with mobile speed of $180 \mathrm{~km} / \mathrm{h}$. Again, it is observed that performance of proposed method I follows the best of two alternative transmission methods by properly configuring the transmission mode within the frame.

Suboptimal to the proposed transmission Method I, the following MIMO transmission mode allocation methods can also be considered.
Method A: Average SNR based MIMO mode allocation with average BER as decision metric: In this method, only one MIMO transmission mode is selected for a frame depending on the average SNR value $\eta$. Specifically, BF is allocated if $\eta<\eta_{\text {th }}$ and SFBC is allocated if $\eta>\eta_{\mathrm{th}}$. This in turn requires finding average BER equations for BF and SFBC. These equations can be obtained by averaging $P_{b}^{\mathrm{bf}}(\tilde{\gamma})$ and $P_{b}^{\mathrm{sfbc}}(\tilde{\gamma})$ over the channel distribution $f_{\tilde{\gamma}}(\tilde{\gamma})$. Then, $\eta_{\mathrm{th}}$ can be calculated from average BER equations. The average BER equations are obtained in Appendix for MISO case. However, closed form solutions to average BER performance is difficult to obtain in a more general MIMO scenario. Performance of Method A corresponds to the better of individual BF and SFBC transmissions.

Method B: Frame-by-frame based mode allocation with quasistatic fading assumption: This method also allocates only one MIMO transmission mode for a frame. The switching decision is made based on BER conditioned on the initial channel knowledge, $\mathbf{H}_{-\tau}$. However, this method ignores channel variation inside the frame. In other words, it is assumed that channel stays the same throughout the frame. Since BF is always better than SFBC when there is perfect channel knowledge, this method always allocates BF. Therefore it can be considered to be suboptimal to the proposed transmission method I when the channel variations cannot be ignored during one frame duration. Performance of Method B corresponds to individual BF transmission.

As can be observed from the Figures 2 and 3, the proposed Method I performs better than Method B by taking channel variations into consideration. It can also be seen that the proposed Method I gives better performance than Method A and this performance is achieved without resorting to average BER performance equations.

\section{B. Proposed Method II: MIMO Transmission Mode Allocation and Bit Loading Based on Average BER}

In this section, we present performance advantage of statistical bit loading when applied on top of the proposed Method I. Statistical bit loading algorithm introduced in Section IV makes use of better channel conditions by transmitting with higher data rate. We expect to see increased advantage of statistical bit loading when number of transmit antennas are higher than the number of receive antennas due to larger antenna array gain. For a given $n_{t}$, array gain is maximized when $n_{r}=1$ as long as channel information is available at the BS. Therefore, MISO configuration has practical importance if one aims at exploiting channel knowledge at the transmitter. We present performance of statistical bit loading algorithm in AR1 channel model for $2 \times 1$ and $4 \times 1$ MISO systems in Figures 4 to 7 . In all of these simulations, signal is transmitted with 4-QAM modulation (for schemes without bit loading), normalized Doppler frequency is fixed at $f_{d} T_{s}=0.044$ and the number of OFDM symbols in a frame is 14. Modulation order and MIMO transmission mode for each symbol are determined based on average BER metrics derived in (21) and (22). First, MIMO transmission mode is determined from average BER expressions. Then, same equations are used in (18) to determine modulation order for each symbol. 


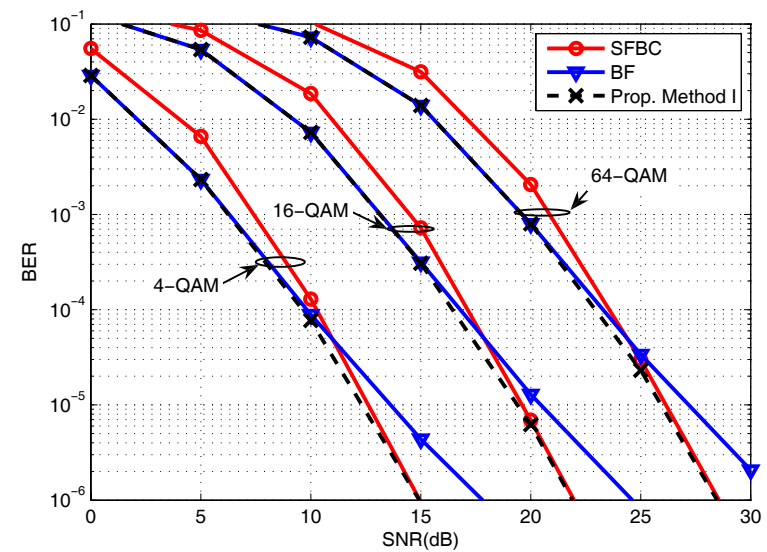

Fig. 2. Average BER performance the proposed method I for uncoded $M$-QAM modulation, $n_{t}=2, n_{r}=2, f_{d} T_{s}=0.044$, number of symbols in a frame $=14$, number of channel taps $d_{f}=4$.

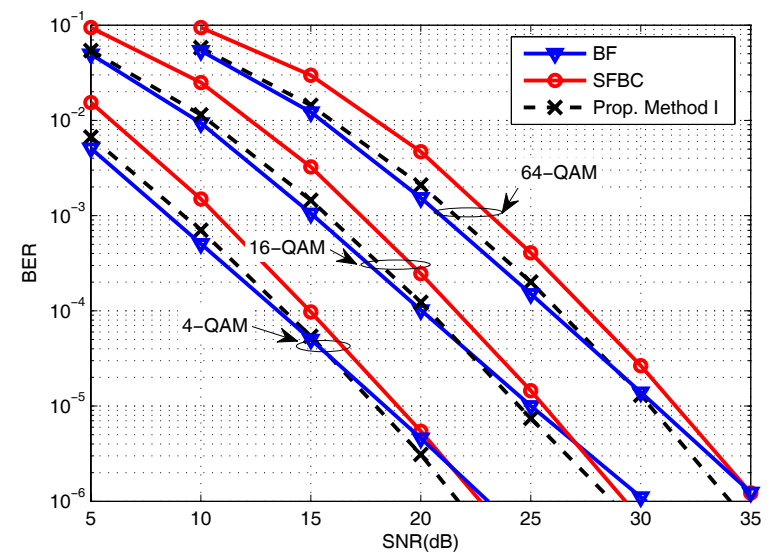

Fig. 3. Average BER performance the proposed method I for uncoded $M$ QAM modulation, $n_{t}=2, n_{r}=2$, mobile speed $=180 \mathrm{~km} / \mathrm{h}$, number of symbols in a frame $=14$, WINNER channel model, NLOS macro rural scenario.

Figures 4 and 5 show the performance for $2 \times 1$ system with frequency diversity orders $d_{f}=1$ and $d_{f}=4$, respectively. Note that the proposed Method II in $2 \times 1$ system does not provide any performance gain over the proposed Method I when there is no frequency diversity. However, there is some gain in higher SNR values for frequency diversity order $d_{f}=4$, as can be seen in Figure 5. In Figures 6 and 7, performances of different transmission methods are shown for $4 \times 1$ system for frequency diversity orders $d_{f}=1$ and $d_{f}=4$, respectively. From Figure 6 , similar to the $n_{t}=2$ case, it is also observed that the proposed Method II in $4 \times 1$ system does not perform better than the proposed Method I in the absence of frequency diversity for $n_{t}=4$. Figure 7 shows performance improvement when frequency diversity is increased to 4 . From these figures, we can see that statistical bit loading algorithm makes effective use of antenna array gain and frequency diversity gain whenever they are available. In Figure 8, we show effect of feedback delay on the performance. In this result, feedback delay of 2 symbol durations is introduced. Performance of the system with feedback delay is shown with dashed lines, whereas the

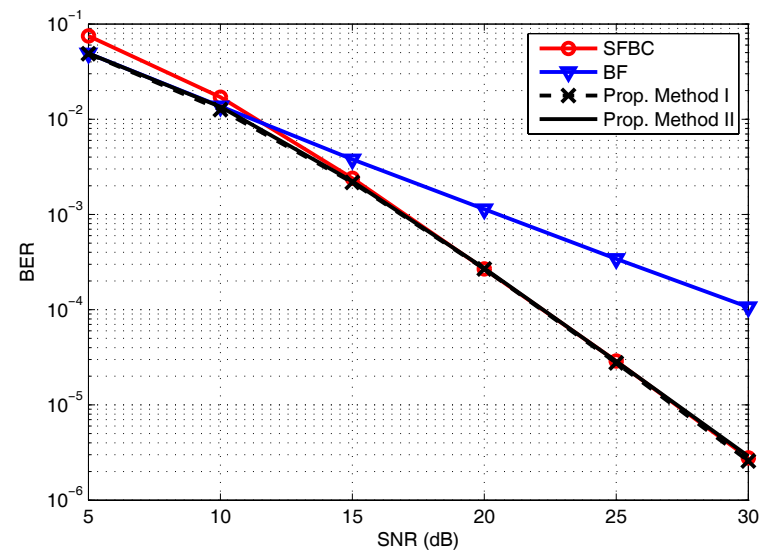

Fig. 4. Average BER performance of the proposed method II, $n_{t}=2, n_{r}=1$, $f_{d} T_{s}=0.044$, number of symbols in a frame $=14, R=28$ bits/frame, $r_{\max }=6$ bits/symbol, number of channel taps $d_{f}=1$.

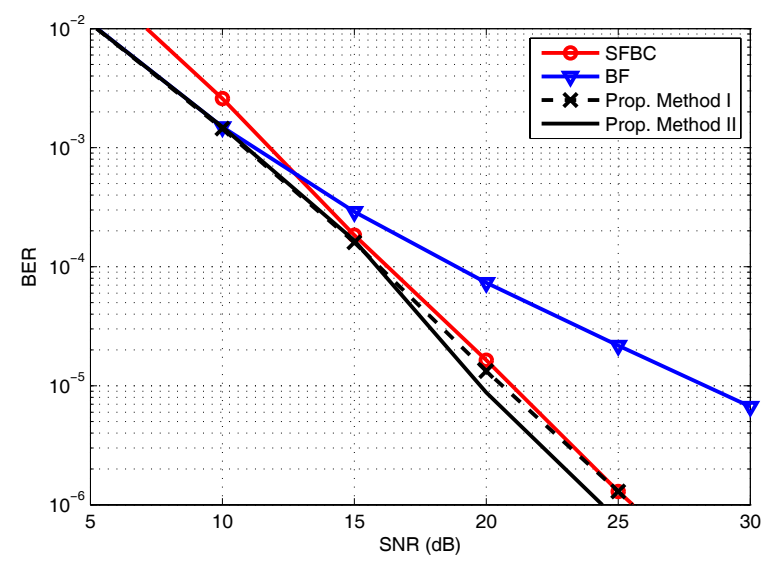

Fig. 5. Average BER performance of the proposed method II, $n_{t}=2, n_{r}=1$, $f_{d} T_{s}=0.044$, number of symbols in a frame $=14, R=28 \mathrm{bits} / \mathrm{frame}, r_{\max }=6$ bits/symbol, number of channel taps $d_{f}=4$.

performance without feedback delay is shown with solid lines. It can be seen that performance of SFBC is not much affected because channel knowledge here is used only for channel selection. As expected, there is some degradation on the performance of $\mathrm{BF}$ and proposed method II. Proposed method II still shows performance gain with respect to both SFBC and BF schemes. Next, we show performance of different transmission methods for $4 \times 1$ system with respect to different normalized Doppler frequencies, $f_{d} T_{s}$, in Figure 9. As can be seen, the proposed method I performs as good as or better than the better of individual BF and SFBC transmissions. However, Method II performs better than individual BF and SFBC transmission for all normalized Doppler speeds. In Table I, ranges of normalized Doppler frequencies with respect to different mobile speeds are presented for the next generation wireless systems. OFDM symbol durations $\left(T_{s}\right)$ of Wimax and LTE systems are used for calculating the corresponding normalized maximum Doppler frequency. Carrier frequencies are also selected for the most common deployment scenarios for each system. If the carrier frequency is higher, then the effects of mobility will be more dramatic. By looking at the 


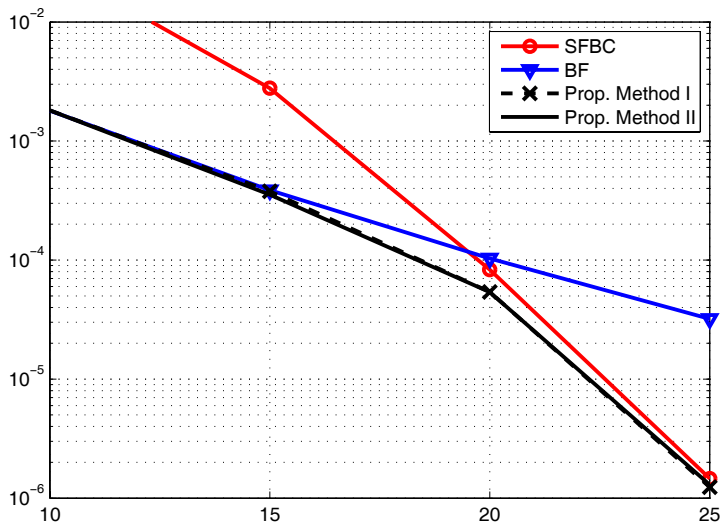

Fig. 6. Average BER performance of the proposed method II, $n_{t}=4, n_{r}=1$, $f_{d} T_{s}=0.044$, number of symbols in a frame $=14, R=28$ bits/frame, $r_{\max }=6$ bits/symbol, number of channel taps $d_{f}=1$.

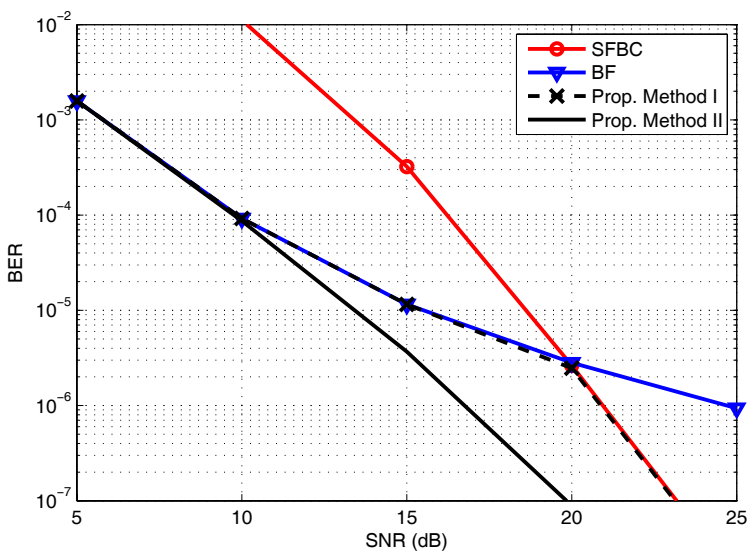

Fig. 7. Average BER performance of the proposed method II, $n_{t}=4, n_{r}=1$, $f_{d} T_{s}=0.044$, number of symbols in a frame $=14, R=28$ bits/frame, $r_{\max }=6$ bits/symbol, number of channel taps $d_{f}=4$.

Figure 9 and range of $f_{d} T_{s}$ values from Table I, advantage of adapting MIMO transmission mode and modulation order in each symbol is apparent in higher vehicular speeds. Note that the number of symbols in one frame is 14 in the simulation results shown in Figure 9. Therefore, $f_{d} T_{f}=14 f_{d} T_{s}$ in our simulations. Figure 10 shows performance of bit loading algorithm for the WINNER channel model in NLOS rural macro environment. The mobile speed is set at $180 \mathrm{~km} / \mathrm{h}$. Since we do not have average BER equations for general channel model, this bit loading algorithm uses Equations (12) and (15) in (18). The performance advantage of proposed method II is also shown for this channel model. Reduced performance gain of proposed method II with respect to fixed transmission mode schemes is due to the decreased frequency selectivity of the channel. Keeping the channel conditions as the same, we introduce feedback delay of 2 OFDM symbol durations to the system in Figure 11. Advantage of proposed method II is still observed with respect to BF and SFBC schemes.

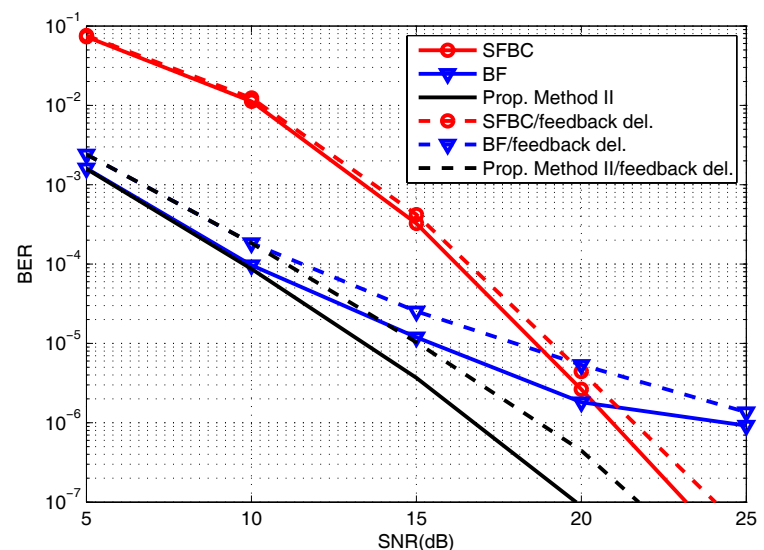

Fig. 8. Average BER performance of the proposed method II with feedback delay, $n_{t}=4, n_{r}=1, f_{d} T_{s}=0.044$, number of symbols in a frame $=14, R=28$ bits/frame, $r_{\max }=6$ bits/symbol, number of channel taps $d_{f}=4$.

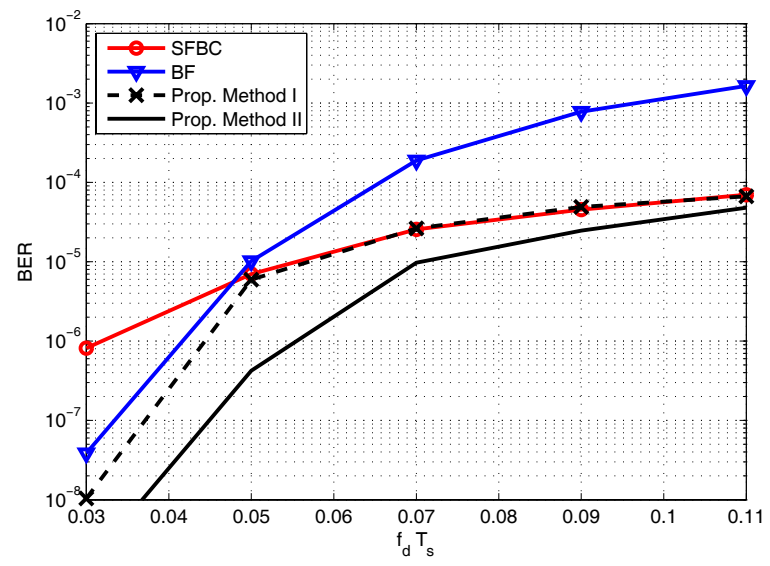

Fig. 9. Average BER performance of the proposed method II, $n_{t}=4, n_{r}=1$, average $\mathrm{SNR}=20 \mathrm{~dB}$, number of symbols in a frame $=14, R=28 \mathrm{bits} / \mathrm{frame}$, $r_{\max }=6$ bits/symbol, number of channel taps $d_{f}=4$.

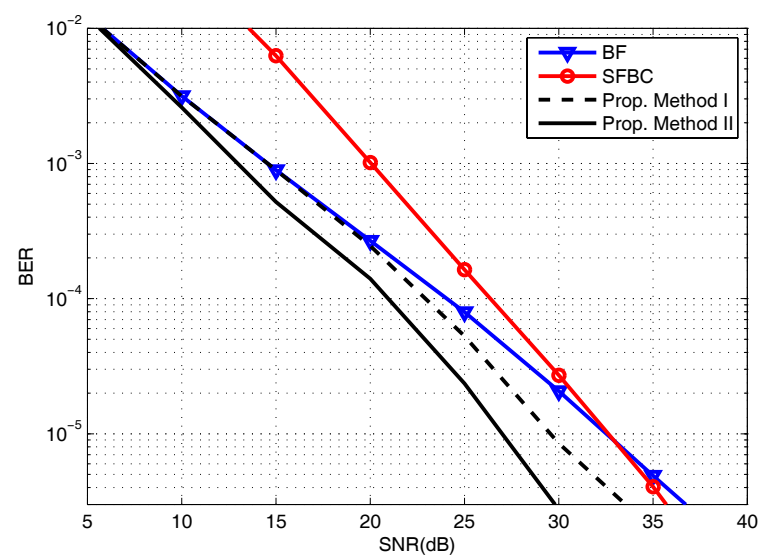

Fig. 10. Average BER performance of the proposed method II, $n_{t}=4$, $n_{r}=1$, mobile speed $=180 \mathrm{~km} / \mathrm{h}$, number of symbols in a frame $=14, R=28$ bits/frame, $r_{\max }=6$ bits/symbol, WINNER channel model, NLOS macro rural scenario. 


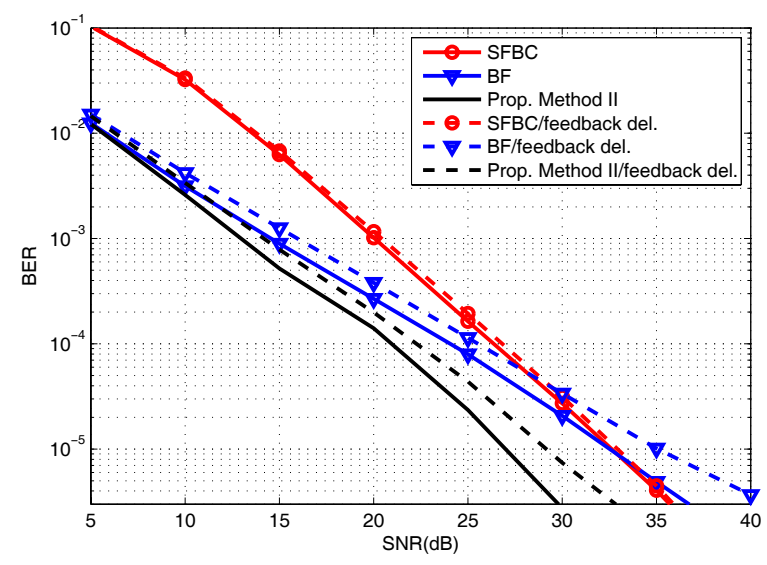

Fig. 11. Average BER performance of the proposed method II with feedback delay, $n_{t}=4, n_{r}=1$, mobile speed $=180 \mathrm{~km} / \mathrm{h}$, number of symbols in a frame $=14, R=28$ bits/frame, $r_{\max }=6$ bits/symbol, WINNER channel model, NLOS macro rural scenario.

\section{CONCLUSIONS}

We have proposed two MIMO transmission strategies for fast fading channels in which quasi-static fading assumption does not hold. In the proposed approach, alternative MIMO transmission modes are allocated for a given frame by taking channel variations into consideration. Specifically, BF is allocated as long as the channel knowledge is reliable at the BS and then transmission is switched to SFBC. The first proposed method makes effective use of initial channel knowledge by assigning transmission modes based on BER performance metrics conditioned on the initial channel knowledge. The method has been shown to perform as good as or slightly better than the best of BF-only and SFBC-only transmissions. In this method, optimization pertains to minimizing BER for a fixed data rate per symbol. Next, we relaxed fixed rate requirement per symbol by allowing different modulation orders across symbols in a given frame. A statistical bit loading algorithm is proposed to take advantage of statistically better channel conditions at the beginning of the frame. Data rate as well as MIMO transmission mode are varied across symbols for a fixed data rate per frame. Simulation results show that the proposed bit loading algorithm provides further performance improvement over the first proposed method. The performance gain increases for increased array gain and for higher frequency diversity orders.

\section{APPENDIX}

In this appendix, we provide derivations of average BER expressions that are used for bit loading scheme for MISO system when channel is uncorrelated across antennas. Note that we use (12) and (15) in (18) for general channel conditions since unconditional BER expressions for a general channel model is not available. In the following we derive unconditional BER metrics for BF and SFBC with multiuser diversity exploitation for spatially uncorrelated channels.

\section{A. Derivation of average BER expressions for MISO case}

We can first rewrite conditional BER expression of BF from (12) for $n_{r}=1$ and uncorrelated antennas, i.e. $\mathbf{R}_{\mathrm{tx}}=\mathbf{I}_{n_{t}}$ as

$$
\begin{aligned}
P_{b}^{\mathrm{bf}}\left(n, M_{n}, \tilde{\gamma}\right) & \approx 0.2\left(1+\frac{1.5 \eta\left(1-\rho_{n}^{2}\right)}{M_{n}-1}\right)^{-1} \\
& \times \exp \left(-\frac{1.5 \rho_{n}^{2} \tilde{\gamma}}{\left(M_{n}-1\right)+1.5 \eta\left(1-\rho_{n}^{2}\right)}\right)
\end{aligned}
$$

Therefore, average BER can be found by integrating over the distribution of initial channel $\tilde{\gamma}$ as

$$
\begin{aligned}
\mathrm{P}_{b}^{\mathrm{bf}}\left(n, M_{n}\right) \approx & \int_{0}^{\infty} 0.2\left(\frac{1.5 \eta\left(1-\rho_{n}^{2}\right)}{\left(M_{n}-1\right)}+1\right)^{-1} \\
& \times \exp \left(-\frac{1.5 \rho_{n}^{2} \tilde{\gamma}}{\left(M_{n}-1\right)+1.5 \eta\left(1-\rho_{n}^{2}\right) \eta}\right) f_{\tilde{\gamma}}(\tilde{\gamma}) d \tilde{\gamma} \\
= & 0.2\left(\frac{1.5 \eta\left(1-\rho_{n}^{2}\right)}{\left(M_{n}-1\right)}+1\right)^{-1} \phi_{\tilde{\gamma}}(s)
\end{aligned}
$$

where $s=\frac{1.5 \rho_{n}^{2}}{\left(M_{n}-1\right)+1.5 \eta\left(1-\rho_{n}^{2}\right)}$. Similarly for SFBC, we can write from (15)

$$
\begin{aligned}
P_{b}^{\mathrm{sfbc}}\left(n, M_{n}, \tilde{\gamma}\right) \approx & 0.2\left(1+\frac{1.5 a \eta\left(1-\rho_{n}^{2}\right)}{n_{t}\left(M_{n}-1\right)}\right)^{-n_{t}} \\
& \times \exp \left(-\frac{1.5 \rho_{n}^{2} \tilde{\gamma}}{\left(M_{n}-1\right)+\frac{1.5 a \eta\left(1-\rho_{n}^{2}\right)}{n_{t}}}\right) .
\end{aligned}
$$

We can write the average BER as

$$
\begin{aligned}
\mathrm{P}_{b}^{\mathrm{sfbc}}\left(n, M_{n}\right) \approx & \int_{0}^{\infty} 0.2\left(1+\frac{1.5 a \eta\left(1-\rho_{n}^{2}\right)}{n_{t}\left(M_{n}-1\right)}\right)^{-n_{t}} \\
& \times \exp \left(-\frac{1.5 \rho_{n}^{2} \tilde{\gamma}}{\left(M_{n}-1\right)+\frac{1.5 a \eta\left(1-\rho_{n}^{2}\right)}{n_{t}}}\right) f_{\tilde{\gamma}}(\tilde{\gamma}) d \tilde{\gamma} \\
= & 0.2\left(1+\frac{1.5 a \eta\left(1-\rho_{n}^{2}\right)}{n_{t}\left(M_{n}-1\right)}\right)^{-n_{t}} \phi_{\tilde{\gamma}}(s)
\end{aligned}
$$

where $s=\frac{1.5 \rho_{n}^{2}}{\left(M_{n}-1\right)+\frac{1.5 a \eta\left(1-\rho_{n}^{2}\right)}{n_{t}}}$. From (21) and (22), we can observe that we need to find the characteristic function of $\tilde{\gamma}, \phi_{\tilde{\gamma}}(s)$ evaluated at $s$. The closed form solution of the characteristic function with $n_{t}$ as a parameter is difficult to obtain. In the following, we provide closed form solutions of $\phi_{\tilde{\gamma}}(s)$ for antenna configurations $n_{t}=2$ and $n_{t}=4$, which are commonly used in practice.

\section{B. Derivation of characteristic function of $\tilde{\gamma}$}

In MISO system, SNR for the selected subchannel is given by $\gamma_{s}=\eta\left\|\mathbf{h}_{s}\right\|^{2}$ for $\mathrm{BF}$ and $\gamma_{s}=\frac{a \eta}{n_{t}}\left\|\mathbf{h}_{s}\right\|^{2}$ for SFBC, where we define $\gamma_{s} \triangleq \beta\left\|\mathbf{h}_{s}\right\|^{2}$. Here, $\beta=\eta$ for $\mathrm{BF}$ and $\beta=\frac{a \eta}{n_{t}}$ for SFBC. When antenna coefficients are uncorrelated, $\gamma_{s}$ is distributed as Chi-square random variable with $2 n_{t}$ degrees of freedom and CDF of $\gamma_{s}$ is given by $F_{\gamma_{s}}\left(\gamma_{s}\right)=1-\exp \left(-\frac{\gamma_{s}}{\beta}\right) \sum_{k=0}^{n_{t}-1} \frac{1}{k !}\left(\frac{\gamma_{s}}{\beta}\right)^{k}$. Therefore, PDF of $\tilde{\gamma}$ can be found from $f_{\tilde{\gamma}}(\tilde{\gamma})=\frac{d F_{\tilde{\gamma}}(\tilde{\gamma})}{d \tilde{\gamma}}=\frac{d\left(F_{\hat{\gamma}_{s}}\left(\hat{\gamma}_{s}\right)\right)^{d}}{d \hat{\gamma}_{s}}$ as

$$
\begin{aligned}
f_{\tilde{\gamma}}(\tilde{\gamma})=d_{f} & {\left[1-\exp \left(-\frac{\tilde{\gamma}}{\beta}\right) \sum_{k=0}^{n_{t}-1} \frac{1}{k !}\left(\frac{\tilde{\gamma}}{\beta}\right)^{k}\right]^{d_{f}-1} } \\
& \times\left[\frac{1}{\beta\left(n_{t}-1\right) !}\left(\frac{\tilde{\gamma}}{\beta}\right)^{n_{t}-1} \exp \left(-\frac{\tilde{\gamma}}{\beta}\right)\right] .
\end{aligned}
$$


For $n_{t}=2, f_{\tilde{\gamma}}(\tilde{\gamma})$ reduces to

$$
\begin{aligned}
f_{\tilde{\gamma}}(\tilde{\gamma})= & d_{f}\left[1-\exp \left(-\frac{\tilde{\gamma}}{\beta}\right) \sum_{k=0}^{1} \frac{1}{k !}\left(\frac{\tilde{\gamma}}{\beta}\right)^{k}\right]^{d_{f}-1} \\
& \times\left[\frac{1}{\beta}\left(\frac{\tilde{\gamma}}{\beta}\right) \exp \left(-\frac{\tilde{\gamma}}{\beta}\right)\right] \\
= & \frac{d_{f}}{\beta} \sum_{k=0}^{d_{f}-1}\left(\begin{array}{c}
d_{f}-1 \\
k
\end{array}\right)(-1)^{k} \frac{\tilde{\gamma}}{\beta}\left(1+\frac{\tilde{\gamma}}{\beta}\right)^{k} \\
& \times \exp \left(-\frac{(k+1) \tilde{\gamma}}{\beta}\right) \\
= & \frac{d_{f}}{\beta} \sum_{k=0}^{d_{f}-1} \sum_{l=0}^{k}\left(\begin{array}{c}
d_{f}-1 \\
k
\end{array}\right)\left(\begin{array}{c}
k \\
l
\end{array}\right)(-1)^{k}\left(\frac{\tilde{\gamma}}{\beta}\right)^{l+1} \\
& \times \exp \left(-\frac{(k+1) \tilde{\gamma}}{\beta}\right) .
\end{aligned}
$$

Characteristic function can be found as

$$
\begin{aligned}
\phi_{\tilde{\gamma}}(s)= & \frac{d_{f}}{\beta} \sum_{k=0}^{d_{f}-1} \sum_{l=0}^{k}\left(\begin{array}{c}
d_{f}-1 \\
k
\end{array}\right)\left(\begin{array}{c}
k \\
l
\end{array}\right)(-1)^{k} \\
& \times \int_{0}^{\infty}\left(\frac{\tilde{\gamma}}{\beta}\right)^{l+1} \exp \left(-\left(\frac{k+1}{\beta}+s\right) \tilde{\gamma}\right) d \tilde{\gamma} \\
= & d_{f} \sum_{k=0}^{d_{f}-1} \sum_{l=0}^{k}\left(\begin{array}{c}
d_{f}-1 \\
k
\end{array}\right)\left(\begin{array}{c}
k \\
l
\end{array}\right)(-1)^{k} \\
& \times \Gamma(l+2)(\beta s+k+1)^{-(l+2)}
\end{aligned}
$$

where $\Gamma($.$) is the Gamma function. We follow similar steps$ to obtain $\phi_{\tilde{\gamma}}(s)$ for $b=4$ as

$$
\begin{aligned}
\phi_{\tilde{\gamma}}(s)= & d_{f} \sum_{k=0}^{d_{f}-1} \sum_{l=0}^{k} \sum_{m=0}^{k-l} \sum_{t=0}^{l}\left(\begin{array}{c}
d_{f}-1 \\
k
\end{array}\right)\left(\begin{array}{c}
k \\
l
\end{array}\right) \\
& \times\left(\begin{array}{c}
k-l \\
m
\end{array}\right)\left(\begin{array}{c}
l \\
t
\end{array}\right)(-1)^{k}\left(\frac{1}{2}\right)^{l+1}\left(\frac{1}{3}\right)^{t+1} \\
& \times(2 l+m+t+3) !(\beta s+k+1)^{-(2 l+m+t+4)} .
\end{aligned}
$$

Equations (24) and (25) can then be plugged into the equations (21) and (22) to obtain average BER expressions. Then, these metrics are used in bit loading scheme as explained in Section IV.

\section{REFERENCES}

[1] S. A. Jafar and A. Goldsmith, "Transmitter optimization and optimality of beamforming for multiple antenna systems," IEEE Trans. Wireless Commun., vol. 3, no. 4, pp. 1165-1175, July 2004.

[2] E. Visotsky and U. Madhow, "Space-time transmit precoding with imperfect feedback," IEEE Trans. Inf. Theory, vol. 47, no. 6, pp. 2632-2638, Sep. 2001.

[3] A. Abdel-Samad, T. N. Davidson, and A.-B. Gershman, "Robust transmit eigen beamforming based on imperfect channel state information," IEEE Trans. Signal Process., vol. 54, no. 5, pp. 1596-1609, May 2006.
[4] M. Payaro, A. Pascual-Inserte, and M. A. Lagunas, "Robust power allocation designs for multiuser and multiantenna downlink communication systems through convex optimization," IEEE J. Sel. Areas Commun., vol. 25, no. 7, Sep. 2007.

[5] G. Jongren and M. Skoglung, "Combining beamforming and orthogonal space-time block coding," IEEE Trans. Inf. Theory, vol. 48, no. 3, pp. 611-627, Mar. 2002.

[6] S. Zhou and G. B. Giannakis, "Optimal transmitter eigen-beamforming and space-time block coding based on channel mean feedback," IEEE Trans. Signal Process., vol. 50, no. 10, pp. 2599-2613, Oct. 2002.

[7] M. K. Simon and M.-S. Alouini, Digital Communications over Fading Channels. Wiley, 2005.

[8] G. L. Turin, "The characteristic function of Hermitian quadratic forms in complex normal random variables," Biometrika, vol. 47, no. 1/2, pp. 199-201, June 1960.

[9] S. Ektabani and H. Jafarkhani, "Combining beamforming and space-time coding using quantized feedback," IEEE Trans. Wireless Commun., vol. 7, no. 3, pp. 898-908, Mar. 2008.

[10] R. Clarke, "A statistical theory of mobile reception," Bell Syst. Tech. J., vol. 47, pp. 957-1000, July 1968

[11] F. K. H. Lee, M. H. Vu, and A. J. Paulraj, "Adaptive vs. diversity transmission for multiuser MISO systems with imperfect CSIT," in Proc. IEEE International Conf. Commun. 2007, pp. 897-901.

[12] I. S. Gradshteyn and I. M. Ryzhik, Table of Integrals, Series and Products, 6th edition. Academic Press, 2000.

[13] A. J. Goldsmith and S.-G. Chua, "Variable-rate variable-power M-QAM for fading channels," IEEE Trans. Commun., vol. 45, pp. 1218-1230, Oct. 1997.

[14] G. Femenias, "BER performance of linear STBC from orthogonal designs over MIMO correlated Nakagami-m fading channels," IEEE Trans. Veh. Technol., vol. 53, pp. 307-317, Mar. 2004.

[15] L. Liu and H. Jafarkhani, "Successive transmit beamforming algorithms for multiple-antenna OFDM systems," IEEE Trans. Wireless Commun., vol. 6, no. 4, pp. 1512-1522, Apr. 2007.

[16] H. Zhang, S. Wei, G. Ananthaswamy, and D. L. Goeckel, "Adaptive signaling under statistical measurement uncertainty in wireless communications," Proc. IEEE, vol. 95, no. 12, pp. 2337-2353, Dec. 2007.

[17] P. Kyösti, et al., "WINNER II channel models," IST-WINNER D1.1.2, ver 1.1, Sep. 2007.

[18] WP5D, "Draft report on requirements related to technical performance for IMT-Advanced radio interface(s) [IMT.TECH]," Working Party 5D Sub-Working Group Radio Aspects, 2nd Meeting, Dubai, United Arab Emirates, July 2008.

[19] 3GPP, Long-Term Evolution (LTE), "Physical Channels and Modulation” (Release 8), 3GPP TS 36.211 v1.2.0, June 2007.

[20] "IEEE 802.16e, "Air Interface for Fixed and Mobile Broadband Wireless Access Systems."

[21] 3GPP, LTE-Advanced, "Requirements for further advancements for EUTRA" (Release 8), 3GPP TR 36.913 v8.0.1, Mar. 2009.

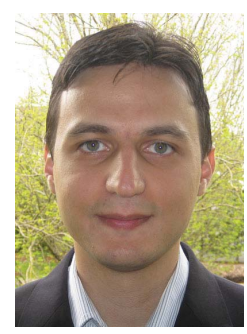

Balkan Kecicioglu received his B.Sc degree from Middle East Technical University (METU), Ankara, Turkey, in 2002. He earned his M.Sc. and Ph.D. degrees from the University of Texas at Dallas, Richardson, Texas in 2011, all in electrical engineering. He spent the summer of 2007 as a graduate intern in Intel Corporation's Wireless Systems Laboratory, Hillsboro, Oregon. In the summer of 2010, he worked as a research intern in Docomo USA Communications Laboratories, Palo Alto, California. His research interests include wireless communications, signal processing for MIMO systems, heterogeneous networks.

Hlaing Minn See page 2264, vol. 58, no. 8, Aug. 2010, of this journal.

Chia-Chin Chong See page 1378, vol. 56, no. 8, Aug. 2008, of this journal. 